\title{
Modular Architecture of Bacterial RNase P Ribozymes as a Structural Platform for RNA Nanostructure Design
}

\author{
Yuri Nozawa, Megumi Hagihara, Shigeyoshi Matsumura, and Yoshiya Ikawa*
}

\begin{abstract}
Ribonuclease $\mathrm{P}(\mathrm{RNase} \mathrm{P})$ is a class of enzymes involved in the processing of precursor tRNAs to remove their 5 '-leader sequences. Ribonuclease $P$ enzymes are classified into two completely distinct classes, i.e. an RNA-based enzyme and a protein-only enzyme. The RNA-based enzyme functions as a ribozyme in which the catalytic machinery is supported by its RNA component consisting of a single RNA molecule. Bacterial RNase $P$ RNAs are a classical class of ribozymes and their structures and catalytic mechanisms have been studied extensively. The bacterial RNase P ribozyme has a modular tertiary structure consisting of two large domains, each of which can self-fold without the partner domain. Such modular architecture, identification of which provided important insight into the function of this ribozyme, is attractive as a structural platform to design functional RNA nanostructures. The first section of this article briefly summarizes the diversity of RNase $P$ mainly focusing on RNA-based enzymes. The second section describes the structures of bacterial RNase $\mathrm{P}$ ribozymes from the viewpoint of their application as modular tools in RNA nanostructure design. The last section summarizes the current state and next steps in modular engineering of RNase P RNAs, including possible design of RNase P ribozyme-based nanostructures.
\end{abstract}

Keywords: Ribozyme $\cdot$ RNA enzyme $\cdot$ RNA nanostructure $\cdot$ RNase $P \cdot$ tRNA processing $\cdot$ Transfer RNA

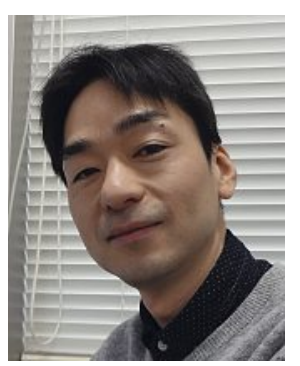

\section{Yoshiya Ikawa}

joined the group of Prof. Donald Hilvert at the ETH Zurich in 2003-2004 as a visiting researcher. He became an associate professor (2004-2013) at Kyushu University (Fukuoka, Japan), and since 2013 he is a professor of biofunctional chemistry at University of Toyama (Toyama, Japan). His research interests include structural aspects of RNA molecules with catalytic and receptor functions. He is also interested in RNA nanotechnology to design self-assembled RNA nanostructures in which naturally occurring RNA structures are employed as building blocks. In this special issue he gives an overview on ribonuclease P (RNase P) RNAs from the viewpoint of their possibility as building blocks for RNA nanostructure design.

\section{Introduction}

Transfer RNAs (tRNAs) are a class of functional RNA molecules that play critical roles as adaptor molecules in translation of genetic information recorded as sequences of nucleic acids to polypeptide sequences. The functions of tRNA molecules depend on their common L-shaped tertiary structure, in which the 3'-ends of the tRNAs are precisely terminated with 5'-CCA-3' to join amino acids through ester bonds.
To produce mature tRNA molecules without extra sequences in both the 3' and 5' regions, primary transcripts of tRNA molecules must be processed at their 5'-leader and 3'-trailer sequence precisely by RNA processing enzymes. ${ }^{[1,2]}$ Ribonuclease $\mathrm{P}$ (RNase P) is a class of enzymes responsible for removal of the 5'-leader sequences from precursor tRNAs (pre-tRNAs) (Fig. 1A).

RNase P enzymes are a class of RNAbased enzymes (ribozymes). RNA-based

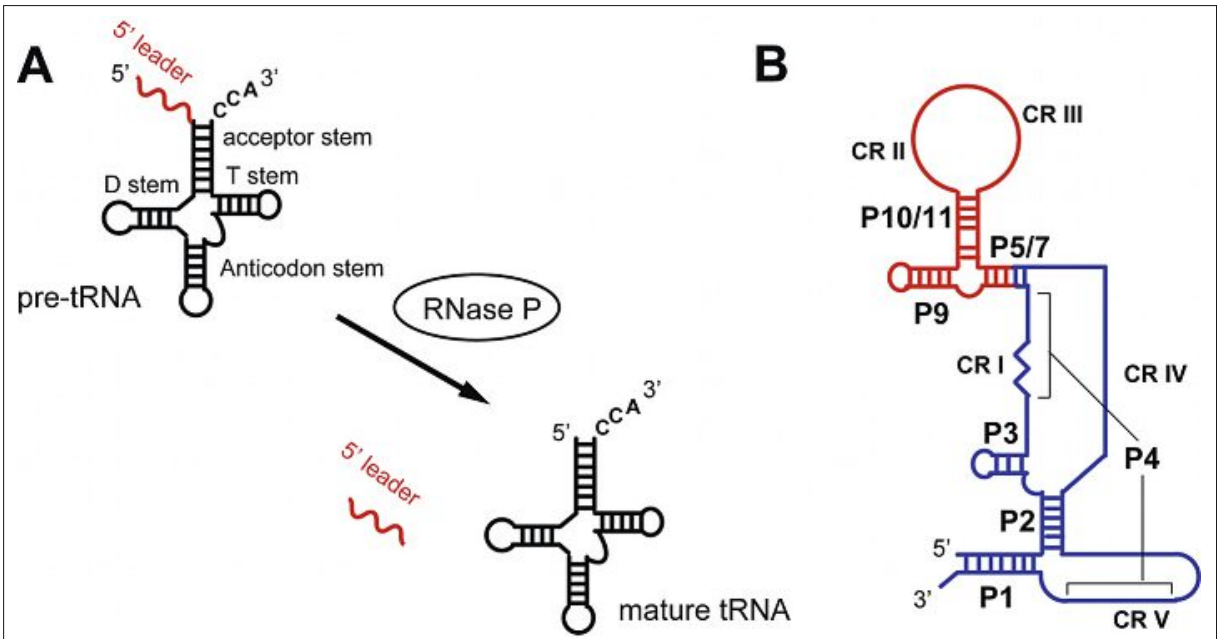

Fig. 1. Reaction catalyzed by RNase $P$ RNAs that have a common secondary structure. A) Endonucleolytic cleavage of pre-tRNA catalyzed by RNase P enzymes. B) Consensus secondary structure of bacterial, archaeal, and eukaryotic nuclear RNase P RNAs. CR I-CR V indicate five conserved regions reported by Chen and Pace. ${ }^{[3]}$ In bacterial RNase P RNAs, regions shown in red and blue form the specificity (S-) domain and catalytic (C-) domain, respectively.

\footnotetext{
${ }^{\star}$ Correspondence: Prof. Y. Ikawa

Department of Chemistry

Graduate School of Science and Engineering

University of Toyama

Gofuku 3190, Toyama, Japan

E-mail: yikawa@sci.u-toyama.ac.jp
} 
RNase $\mathrm{P}$ enzymes commonly contain a single RNA molecule that can form a modular secondary structure (Fig. 1B). ${ }^{[3]}$ Escherichia coli RNase P enzyme was purified and characterized by Sidney Altman and his co-workers. They found that RNA components of bacterial RNase P (RNase $P$ RNA) efficiently cleaved pre-tRNAs without protein components under highionic buffer conditions. ${ }^{[4]}$ The discovery of the catalytic function of bacterial RNase P RNA earned Altman the 1989 Nobel Prize in Chemistry, which was shared with Thomas R. Cech who discovered the selfsplicing activity of group I intron RNAs. Their discoveries opened up a new research field of catalytic functions of RNA molecules. Bacterial RNase P consists of a single large RNA (typically ca. 300-400 nucleotides), which plays a catalytic role, and a single small protein (ca. 120 amino acids) that supports the RNA-based catalysis. ${ }^{[4,5]}$ Archaeal RNase P enzymes generally possess four proteins, although archaeal RNase P RNAs play catalytic roles. ${ }^{[6]}$ Some archaeal RNase P RNAs showed catalytic ability without protein components, but their activities are more closely dependent on the protein components than the bacterial enzymes. ${ }^{[6,7]}$ Eukaryotic RNase $P$ enzymes are more complex than bacterial and archaeal enzymes due to their protein components, of which there are nine and ten in the nuclear RNase $\mathrm{P}$ enzymes from Saccharomyces cerevisiae and Homo sapiens, respectively. ${ }^{[8]}$ Although full catalytic ability of their RNA components is strongly dependent on the protein components, the H. sapiens RNase P RNA exhibited detectable activity, which was about $10^{6}$-fold lower than that of E. coli RNase P RNA. [9] Comparison of RNA-based RNase P from bacteria, archaea, and eukaryotic nuclei suggested a possible evolutionary process of RNA-based RNase P enzymes as ribonucleoproteins, with gradual substitution of the structural roles of RNA elements with protein components, while the RNA component retained the central roles in catalysis.

Due to the essential roles of RNase $\mathrm{P}$ enzymes in tRNA processing, RNA-based RNase $\mathrm{P}$ enzymes are found in all domains of life. On the other hand, exceptional examples have also been observed. The most extremely exceptional example is seen in the obligate symbiont, Nanoarchaeum, the genome of which contains no genes encoding RNase $\mathrm{P}$ enzymes because its precursor tRNAs have no 5'-leader sequences. ${ }^{[10]}$ Therefore, there is no requirement for RNase $\mathrm{P}$ in biosynthesis. The second exceptional example is seen in some eukaryotic organelles, a few bacteria, and a few archaea, in which RNase P enzymes are composed only of proteins. ${ }^{[11-14]}$ These protein-only RNase P enzymes are desig- nated as PRORPs (protein-only RNase $\underline{\mathrm{P}}$ ). Plant and bacterial PRORPs contain a single polypeptide, while human mitochondrial PRORP is composed of three polypeptides, one of which is a homolog of the single-polypeptide enzymes in plants. ${ }^{[13]}$ It should also be noted that the evolutionary divergence of RNaseP is seen in eukaryotic organelles, in which RNA-based RNase P seems to be missing in plant and human mitochondria but is present in fungal mitochondria. ${ }^{[15]}$ These observations make RNA-based RNase P enzymes attractive materials to study enzyme evolution, including the transition from RNA enzymes to protein enzymes. The common secondary structure of RNA components of RNA-based RNase P suggest that RNase P ribozyme is a relict of the RNA world and had already played a role in tRNA processing in the last universal common ancestor (LUCA). Although the emergence and evolution of PRORPs are less well understood, it was recently reported that some bacteria and archaea possess both RNase P RNA genes and PRORP genes, the gene products of which both exhibited RNase $\mathrm{P}$ enzyme activities. ${ }^{[14]}$

\section{Bacterial RNase $P$ ribozymes}

\subsection{Modular Organization of Bacterial RNase P Ribozymes}

As one of the first RNA enzymes to be discovered, bacterial RNA-based RNase $\mathrm{P}$ has been studied extensively. Among the known naturally occurring ribozymes, RNase P RNA is an exceptional example of a true catalyst with multiple turnover ability. Bacterial RNase P RNAs typically consists of 350-400 nucleotides and show catalytic activity without any protein components. ${ }^{[4]}$ To exhibit full catalytic ability in vivo, bacterial RNase P RNAs form a ribonucleocomplex with a small protein of about $14 \mathrm{kDa}$ typically containing 120 amino acids, ${ }^{[16]}$ which binds to the C-domain of the RNA component. ${ }^{[17]}$ This feature increases the scientific importance of bacterial RNase P as one of the simplest examples of ribonucleoprotein enzymes. The functional and structural roles of the respective elements in the consensus secondary structure of bacterial RNase $\mathrm{P}$ RNAs have been elucidated through extensive biochemical analyses of those from E. coli and Bacillus subtilis as major model ribozymes. ${ }^{[5]}$ Biochemical studies have further progressed by the support of structural studies with RNA crystallography performed mainly using their thermostable homologs. Extensive sequence comparison of bacterial RNase P RNAs and experimental analyses of $E$. coli and B. subtilis RNase $\mathrm{P}$ indicated that bacterial RNase P RNAs can be divided into two major classes based on their secondary structures. E. coli RNase P RNA belongs to the first class, termed A-type, while $B$. subtilis RNase P RNA belongs to the second class, termed B-type (Fig. 2A and 2B). ${ }^{[5]}$ The RNA secondary structures of the two classes share common structural elements organizing the core catalytic region (blue and red elements in Fig. 1B), which directly participates in recognition of pre-tRNAs and the catalytic machinery employing two metal ions to cleave the single phosphodiester bonds in pre-tRNAs. ${ }^{[17,18]}$

The core elements in the secondary structure can be divided into two structural domains designated as the specificity (S) domain and the catalytic (C) domain. In both A-type and B-type RNase P RNAs, the S-domain and C-domain have been shown to fold independently, and the intact ribozyme structures are formed through modular assembly of folded $\mathrm{S}$ and C-domains. ${ }^{[19,20]}$ The S-domain predominantly contributes to pre-tRNA recognition through the interaction with their

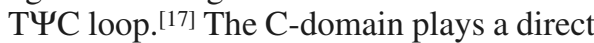
role in hydrolytic cleavage of the 5'-leader sequence by organizing the catalytic machinery. ${ }^{[17,18]}$ In addition to its direct role in catalysis, the $\mathrm{C}$-domain recognizes the acceptor stem and the conserved 3'-CCA of tRNA to place the cleavage site within the catalytic center.[17] The modular organization of RNase $\mathrm{P}$ ribozymes has similarities to that of the group I ribozymes because the group I ribozymes also consist of two modular structural elements (P4-P6 domain and P7-P3-P8 domain), in which the P7-P3-P8 domain provides the core catalytic machinery, ${ }^{[21]}$ whereas the P4-P6 domain supports catalysis through recognition of the 5'-splice site. ${ }^{[22]}$

To stabilize the catalytic core structure as well as two modular domains composing the core, structural elements are extended at the periphery of the core region (Fig. 2).[23] Distinct organization of the peripheral elements in A-type and B-type RNase P RNAs not only differentiates their secondary structures but also affects the biochemical and biophysical properties of the two types of RNAs. The minimal core structure of bacterial RNase P ribozymes was deduced by sequence comparison. ${ }^{[24}$ Biochemical analyses have also been performed to examine the catalytic abilities of truncated forms of RNase P RNAs. ${ }^{[24-26]}$ A simplified form of RNase P RNA consisting of 263 nucleotides was first designed by extracting structural elements common between typical A-type (E. coli) and B-type (Bacillus megaterium) RNAs. The resulting RNase P RNA retained the substrate specificity and catalytic ability of bacterial RNase P ribozymes, and can be regarded as a small and chimeric form of the two types of bacterial RNase 


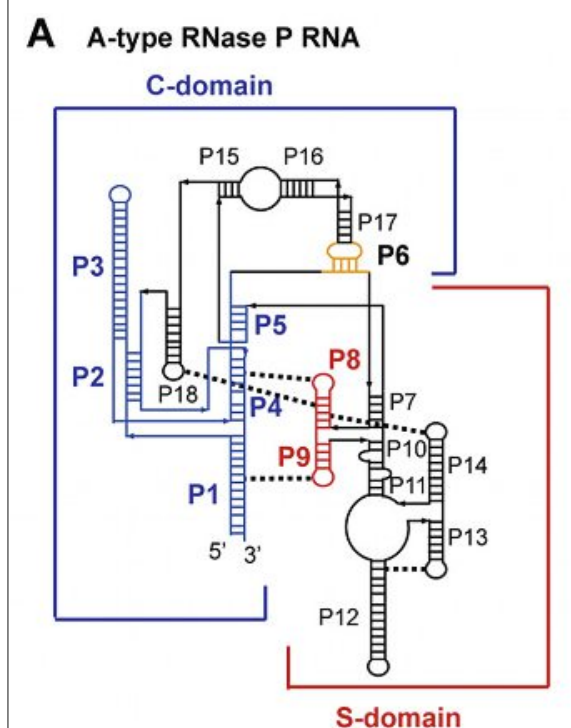

\section{B B-type RNase P RNA}

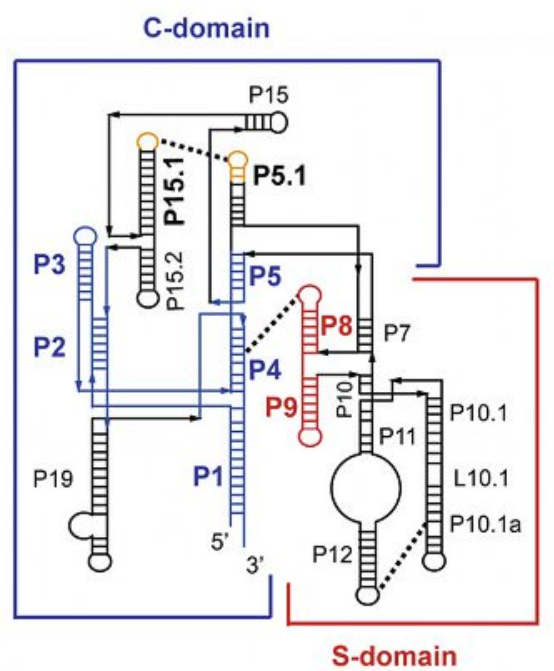

C A-type RNase P RNA

C-domain

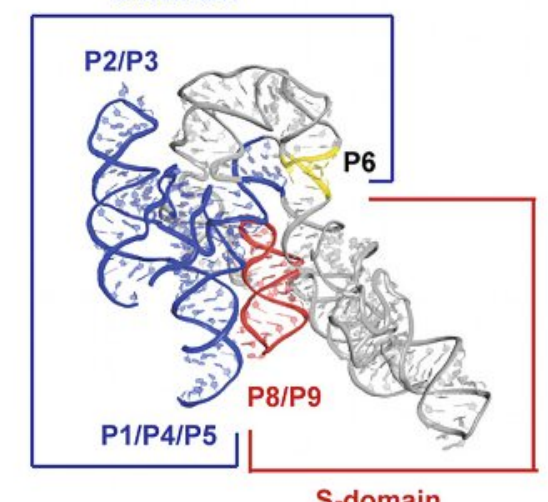

S-domain
D B-type RNase P RNA

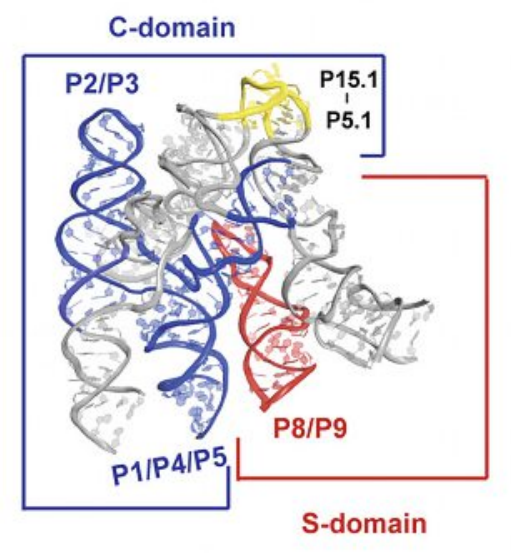

Fig. 2. Two major types of bacterial RNase $P$ ribozymes consisting of two large modular domains. $A$ and B) Consensus secondary structures of A-type (A) and B-type bacterial RNase P RNAs. Both types of structure consist of an S-domain and C-domain, which are covalently connected at the P5 and P7 regions. Regions shown in blue and orange compose a conserved catalytic core. Regions shown in orange are tertiary interactions engineered in their bimolecular derivatives. Thick broken lines indicate tertiary structures contributing to assembly between the S-domain and C-domain. C and D) Three dimensional structures of an A-type RNA from Thermotoga maritima (C) and a B-type RNA from Bacillus stearothermophilus (D).

P RNA. ${ }^{[24]}$ A naturally occurring example of a highly truncated B-type RNA was found in Mycoplasma fermentans consisting of 273 nucleotides. ${ }^{[25]}$ This small B-type RNA was further truncated rationally to yield the Micro P RNA consisting of only 211 nucleotides while retaining ribozyme activity. ${ }^{[25]}$ A naturally occurring example of a highly truncated A-type RNA was also found in archaeal RNase P RNAs. ${ }^{[26]}$ Pyrobaculum aerophilum possesses an A-type like RNase P RNA only 207 nucleotides in length due to the lack of most of the regular S-domain elements. The catalytic activity of this small A-type like RNA to cleave the Pyrobaculum pretRNA was confirmed by in vitro assay.[26] The catalytic ability of the Pyrobaculum RNase P RNA seemed consistent with the results of deletion analyses in which $E$. coli RNase P RNA (A-type) with deletion of the S-domain retained residual catalytic activity. ${ }^{[27,28]}$ To address the ribozyme activity of the B-type C-domain RNA, an in vitro evolution experiment was also performed, ${ }^{[29]}$ in which RNA sequences were successfully identified to serve as artificial substrates for the C-domain RNA from $B$. subtilis RNase P.

\subsection{Three Dimensional (3D) Structures of Bacterial RNase $P$ Ribozymes}

A series of biochemical and phylogenetic studies elucidated the structural organization of both A-type and B-type of bacterial RNase $\mathrm{P}$ ribozymes. These results were further confirmed by structural studies of these ribozymes. The crystal structures of the B-type S-domain RNA from B. subtilis and A-type S-domain RNA from Thermus thermophilus were reported in 2003 and 2004, respectively.[30,31] The 3D-structures of the full-length A-type and B-type RNase P ribozymes were both reported in 2005. ${ }^{[32,33]}$ For crystallization of these ribozymes, thermostable ribozymes (A-type RNA from Thermotoga maritima and B-type RNA from Bacillus stearothermophilus) were employed as alternatives to the E. coli (A-type) and B. subtilis (B-type) RNase P RNAs, respectively (Fig. 2C and 2D). In 2010, the 3D-structure of the T. maritima A-type RNase P holoenzyme was also solved, [17] in which RNase P RNA captured its substrate tRNA and employed the C-domain for association with the protein component.

Comparison of the two types of fulllength ribozymes enabled us to determine the 3D-architecture of the conserved core structure. The conserved core is composed of three helical elements, P1/P4/P5, P2/ P3, and P8/P9 (Fig. 2). Two of the three helical elements (P1/P4/P5 and P2/P3) are provided by the $\mathrm{C}$-domain, while $\mathrm{P} 8 / \mathrm{P} 9$ is from the S-domain. The catalytic site of the ribozyme holding two $\mathrm{Mg}^{2+}$ ions is located within the P4 duplex in the P1/P4/P5 helical domain. ${ }^{[17]}$ This structural feature is consistent with the phylogenetic observation that P4 is the most highly conserved region because 11 of the 21 conserved nucleotides were located in and around P4. ${ }^{[3]}$ The tertiary structures of the conserved core elements, which are common to A-type and B-type RNAs, are stabilized by peripheral elements. Peripheral elements participate in tertiary interactions (Fig. 2), which not only contribute to stabilize independent folding of the S- and C-domains but also play critical roles in stable assembly between $\mathrm{S}$ - and $\mathrm{C}$-domains to maintain the intact core structure. Biochemical and structural analyses identified distinct sets of tertiary interactions between the A-type and B-type RNase P RNAs (Fig. 2).

The A-type RNAs typically utilize six tertiary interactions (Fig. 2A and 2C). ${ }^{[17,23,32]}$ Two of these tertiary interactions contribute to self-folding of the $\mathrm{S}$-domain, the third (P6 base pairs) is critical for self-folding of the C-domain, and the remaining three, one of which is the L8-P4 interaction, are involved in interdomain assembly of the two domains. The B-type RNAs use three tertiary interactions (Fig. 2B and 2D), ${ }^{[23,33]}$ each of which contributes to self-folding of the S-domain, self-folding of the C-domain (P5.1-P15.1 interaction), and L8-P4 interdomain assembly, respectively. The L8-P4 interaction is formed inside the core structure and is conserved between both types of RNase P RNA for assembly of the S- and $\mathrm{C}$-domains. The modular architecture of large ribozymes, such as bacterial RNase $\mathrm{P}$ ribozymes and group I ribozymes, is an important feature in ribozyme biochemis- 
try, including the molecular evolution of RNA structures to perform sophisticated functions.

\subsection{Bimolecular Reconstitution of RNase $P$ Ribozymes through Assembly of the S-domain RNA and C-domain RNA}

The modular structures of large ribozymes are regarded as a promising platform for the design of self-assembled RNA nanostructures, which may be applicable to new classes of biomedical and diagnostic tools. The Tetrahymena group I ribozyme has been used to construct RNA nanostructures with polygonal ribozyme assembly and one-dimensional ribozyme assembly. ${ }^{[34,35]}$ These RNA nanostructures were designed through modular engineering using either of two bimolecular derivatives of this ribozyme $(\mathrm{P} 5 \mathrm{abc}+\Delta \mathrm{P} 5$ bimolecular ribozyme[36] for polygonal assembly or $\mathrm{P} 4 \mathrm{P} 6+\mathrm{P} 3 \mathrm{P} 7$ bimolecular ribozyme ${ }^{37,38]}$ for $1 \mathrm{D}$-array assembly). The two bimolecular derivatives both consist of two modular structural RNAs. The catalytic ability of the parent Tetrahymena ribozyme can be reconstituted through noncovalent assembly of the two structural RNAs. Strategies for structural redesign of bimolecular Tetrahymena ribozymes to generate assembled RNA nanostructures would be applicable to bacterial RNase P ribozymes because they also consist of two structural domains.

Bacterial RNase P ribozymes are commonly composed of two independently folded domains (S- and C-domains) (Fig. 2). Each of the two domains folds into its tertiary structure without assistance from its partner domain. ${ }^{[19,20]}$ These analyses and also structural studies using X-ray crystallography indicated that the S-domain and $\mathrm{C}$-domain are separated physically at the junction of the P7 element and the P5 element. In this dissection, the 5'-strand of P6 element in the A-type RNA and P5.1 element in the B-type RNA belongs to the C-domain (Fig. 3A and 3B). To redesign RNase $\mathrm{P}$ ribozymes in a bimolecular format consisting of separately prepared S-domain RNA and C-domain RNA, the full-length RNase $\mathrm{P}$ ribozyme was first engineered in a circular form by enclosing the ends of the P1 duplex by adding a terminal loop (Fig. 3). The resulting circular RNA was divided into S- and C-domains, each of which consisted of a single RNA strand. ${ }^{[19,20]}$ A bimolecular system in $E$. coli RNase $\mathrm{P}$ ribozyme consisting of the $\mathrm{S}$-domain RNA and C-domain RNA has been examined (Fig. 3A). The self-folding ability of each domain was demonstrated by structural and biochemical studies. Reconstitution of the catalytic ability was also reported, although the physical affinity between the two domains was rather

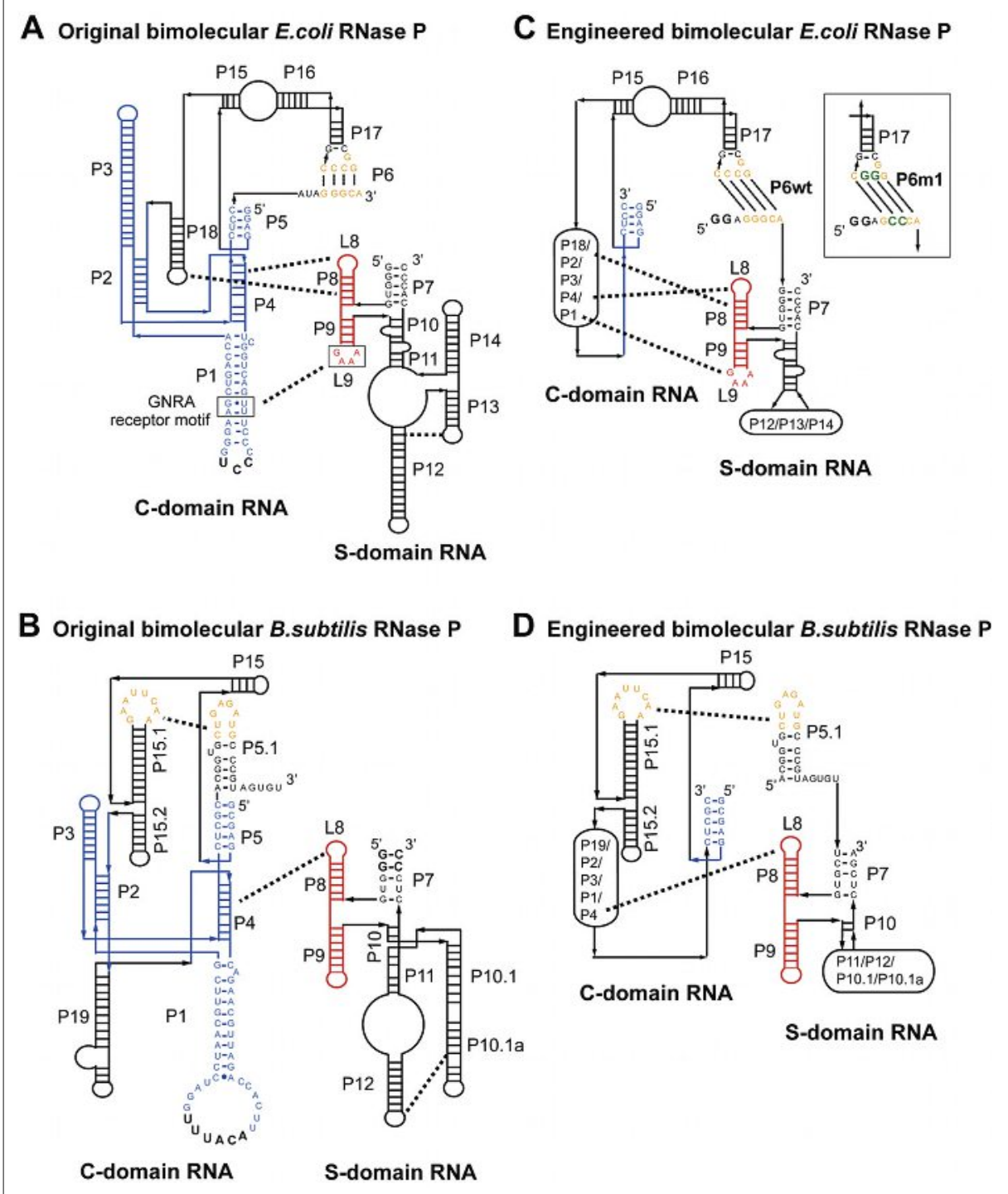

Fig. 3. Bimolecular derivatives of bacterial RNase $P$ ribozymes. A and B) Original versions of bimolecular ribozymes, which were designed according to the structural organization of the S- and C-domains in each RNase P RNA. In the bimolecular format, each domain RNA self-folds without aid from its partner domain RNA. C and D) Engineered versions of bimolecular ribozymes, in which P6 or P15.1-P5.1 interaction supporting the C-domain structures was additionally used as an interdomain interaction to strengthen the assembly of the S- and C-domain RNAs.

weak. ${ }^{[20]}$ We also confirmed the catalytic activity of the bimolecular system in the presence of each domain at $1 \mu \mathrm{M}$ and substrate pre-tRNA at $0.5 \mu \mathrm{M}$ (Fig. $4 \mathrm{~A})$, but its efficiency was quite low (product yield was only $3.3 \%$ after 1 -hour reaction) probably because the concentrations of the two RNA domains were much lower than the reported $K_{\mathrm{d}}$ value of $14 \mu \mathrm{M}$. $^{[20]}$ A bimolecular derivative of $B$. subtilis RNase $\mathrm{P}$ ribozyme (Fig. 3B) showed no detectable activity (Fig. 4A) although its S-domain and C-domain have self-folding ability. This was probably because of the poor association between the two domains, which form only one interdomain tertiary interaction (L8-P4). Thus, their interdomain association may be even weaker than that in the bimolecular E. coli RNase P ribozyme with the three interdomain tertiary interactions (L8-P4, L9-P1, and P8-P18). Assembly of S- and C-domains in the bimolecular
E. coli and B. subtilis RNase P ribozymes is too weak to allow their use as modular tools for RNA nanostructure design. In bimolecular systems of the Tetrahymena ribozyme, association of the two modular RNAs is rather strong (reported $K_{\mathrm{d}}$ values of $25-50 \mathrm{nM}$ in the $\mathrm{P} 4 \mathrm{P} 6+\mathrm{P} 3 \mathrm{P} 7$ format ${ }^{[37]}$ and $0.1 \mathrm{nM}$ in the $\mathrm{P} 5 \mathrm{abc}+\Delta \mathrm{P} 5$ format ${ }^{[39]}$ ).

To improve the utility of the bimolecular RNase $\mathrm{P}$ ribozymes for RNA nanostructure design, the binding affinity between the two domain RNAs must be improved. Therefore, we engineered a tertiary interaction within the $\mathrm{C}$-domain (P6 base pairs in the A-type RNA and P5.1-P15.1 interaction in the B-type) to an interdomain interaction to support the association between the $\mathrm{S}$ - and $\mathrm{C}$-domains (Fig. 3C). Although this modification may disturb the self-folding ability of the C-domain, each tertiary interaction must be regenerated correctly through assem- 


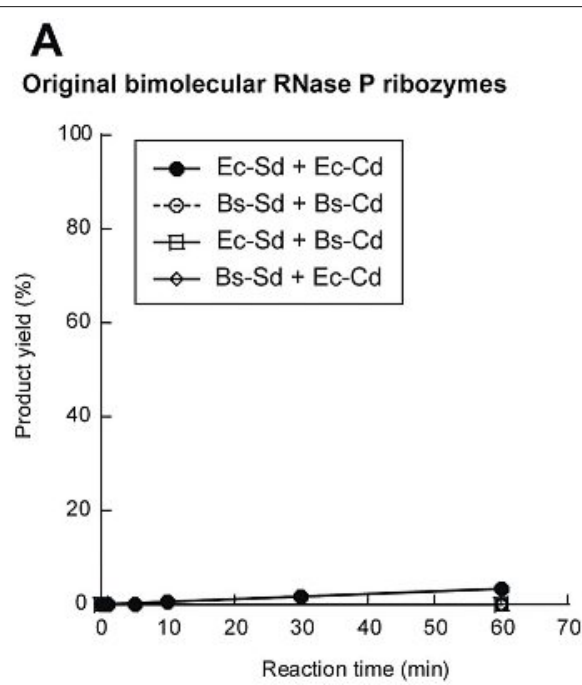

B

Engineered bimolecular RNase $\mathrm{P}$ ribozymes

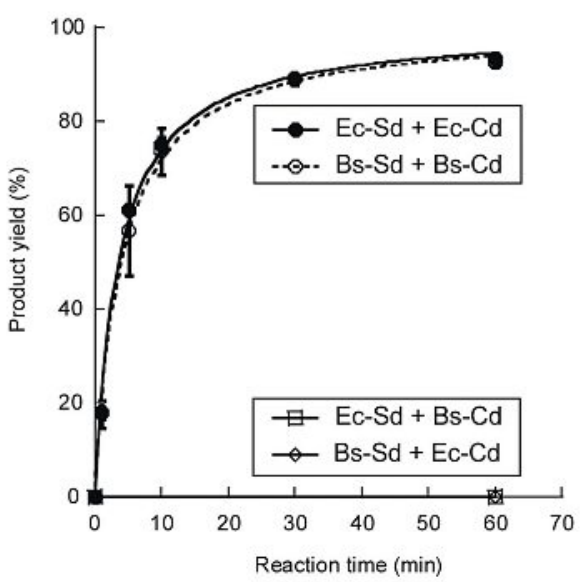

C

Engineered bimolecular E. coli RNase $\mathrm{P}$ ribozymes

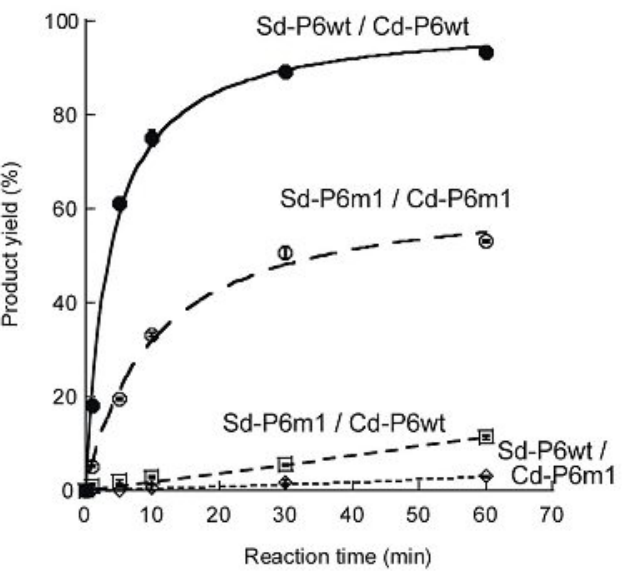

Fig. 4. Site-specific cleavage reactions of a pre-tRNA $A^{\mathrm{Tyr}}$ catalyzed by bimolecular bacterial RNase $\mathrm{P}$ ribozymes. All reactions were performed in the presence of $0.5 \mu \mathrm{M}$ human pre-tRNA ${ }^{\mathrm{Ty}},{ }^{[46]}$ the $3^{\prime}$-end of which was labeled with BODIPY fluorophore, $1.0 \mu \mathrm{M}$ S-domain RNA, and $1.0 \mu \mathrm{M}$ C-domain RNA with $50 \mathrm{mM}$ Tris- $\mathrm{HCl}(\mathrm{pH} 7.5), 50 \mathrm{mM} \mathrm{MgCl}_{2}$, and $1.0 \mathrm{M} \mathrm{KCl}$ at $37^{\circ} \mathrm{C}$. Ec and Bs indicate E. coli and B. subtilis respectively. Sd and Cd indicate $\mathrm{S}$-domain and C-domain respectively. A) Cleavage reactions of a human pre-tRNA $\mathrm{A}^{\mathrm{Tyr}}$ catalyzed by the original versions of bimolecular RNase $\mathrm{P}$ ribozymes consisting of cognate pairs of S- and C-domains and heterologous pairs of the two domains. B) Cleavage reactions of a pre-tRNA $A^{\text {Tyr }}$ catalyzed by the engineered versions of bimolecular RNase P ribozymes consisting of cognate pairs of S- and C-domains and heterologous pairs of the two domains. C) The P6 element in the engineered $E$. coli bimolecular ribozyme governs the catalytic activity that is dependent on assembly of the Sand C-domains.

bly of the two modified domains. In the $B$. subtilis RNase P ribozyme, a catalytically active bimolecular format has already been reported by appending the P5.1 element to the S-domain (Fig. 3D). ${ }^{[19]}$ Under our assay conditions, we also confirmed that this molecular design markedly improved the activity of the bimolecular B. subtilis RNase P ribozyme (Fig. 4B). The catalytic activity of the bimolecular E. coli RNase $\mathrm{P}$ ribozyme also improved significantly (Fig. 4B). The binding specificity between cognate partners is also an important factor in modular assembly of the domain RNAs. Therefore, we examined the activities of bimolecular ribozymes with heterologous pairs of S- and C-domains. In both original and engineered systems, heterologous $\mathrm{S}$ - and C-domain pairs showed no detectable activity (Fig. 4A and 4B), suggesting that the E. coli and B. subtilis bimolecular ribozymes may be used as orthogonal pairs of RNA modular assembly units in designing multi-component RNA nanostructures.

In engineered bimolecular RNase $\mathrm{P}$ ribozymes, the P6 element in the bimolecular E. coli ribozyme is attractive as a modular unit because the binding specificity between $\mathrm{S}$ - and $\mathrm{C}$ domains may be programmable through the identity of P6 base pairs. ${ }^{[40]}$ Therefore, we designed a variant pair of engineered bimolecular $E$. coli ribozymes with variant P6 base pairs (P6m1) (Fig. 3C). Although the P6m1 variant showed only half the activity of the parent bimolecular ribozyme, two bimolecular ribozymes with mismatched P6 base pairs showed much less activity than the parent ribozyme and the P6m1 variant (Fig. 4C). The P6 base pairs can there- fore be used as elements to install binding specificity to the assembly of the S- and $\mathrm{C}$-domains in the A-type bimolecular ribozyme. The A-type RNase P ribozyme possesses a tertiary interaction between L9 and $\mathrm{P} 1$ elements as an interdomain interaction to assemble the $\mathrm{S}$ - and $\mathrm{C}$-domains. The L9-P1 interaction is mediated by a GNRA-receptor interaction. Such GNRAreceptor interactions constitute a class of tertiary interacting motifs the binding specificity of which is programmable. ${ }^{[41,42]}$ Although experimental validation is needed, combinatorial use of P6 base pairs and L9-P1 interaction seems promising as a means to improve programmable assembly between the S- and C-domains of the A-type RNAs. A similar set of interactions (base pair interaction and GNRA-receptor interaction) has been successfully employed for the programmable formation of RNA nanostructures based on the bimolecular Tetrahymena group I ribozyme. [34] To determine the physical affinity between $\mathrm{S}$ - and C-domains in the parent and modified versions of bimolecular RNase P ribozymes, we performed electrophoresis mobility shift assay to detect bimolecular complexes. Under the assay conditions (1.0 $\mu \mathrm{M}$ of each RNA component) that resembled those for analysis of the $\mathrm{P} 5 \mathrm{abc}+\Delta \mathrm{P} 5$ bimolecular Tetrahymena ribozyme, ${ }^{[43]}$ stable complexes were hardly detected even with the engineered version of the E. coli RNase P ribozyme (data not shown). This result suggests that further improvement may be needed to achieve utility of bimolecular RNase P ribozymes comparable to that of the bimolecular Tetrahymena ribozymes.

\section{Conclusion}

This article presented a brief overview of the ribonuclease $\mathrm{P}$ ribozymes, especially focusing on bacterial enzymes that act as catalytic RNA molecules. Modular architecture and structural diversity of their 3D-RNA structures make them attractive as a platform RNA structure for designing RNA nanostructures. Comparison of two versions of biomolecular RNase P ribozymes from $E$. coli and $B$. subtilis indicated that conversion of the P6 base pairs in the A-type ribozyme and P5.1-P15.1 interaction in the B-type ribozyme, both of which originally serve as interactions within the S-domain, to interdomain interactions improved the assembly of the $\mathrm{S}$ - and C-domain RNAs to conduct the catalytic activity. In the A-type RNase P ribozymes, the engineered bimolecular ribozymes seem promising because the P6 base pairs and possibly the L9-P1 interaction can be used to program binding specificity between the S- and C-domains. A possible nanostructure design based on the engineered bimolecular RNase P is shown in Fig. 5.

In this design, an assembly unit composed of two RNA strands was designed by covalently connecting the P12 element of the S-domain and P3 element of the C-domain. The resulting unit RNA was inactive but capable of forming the bimolecular ribozyme unit through intermolecular assembly of the unit to form a one-dimensional assembly of RNase P structures. However, the engineered E. coli bimolecular ribozyme is not directly applicable to the design and characterization of RNA 


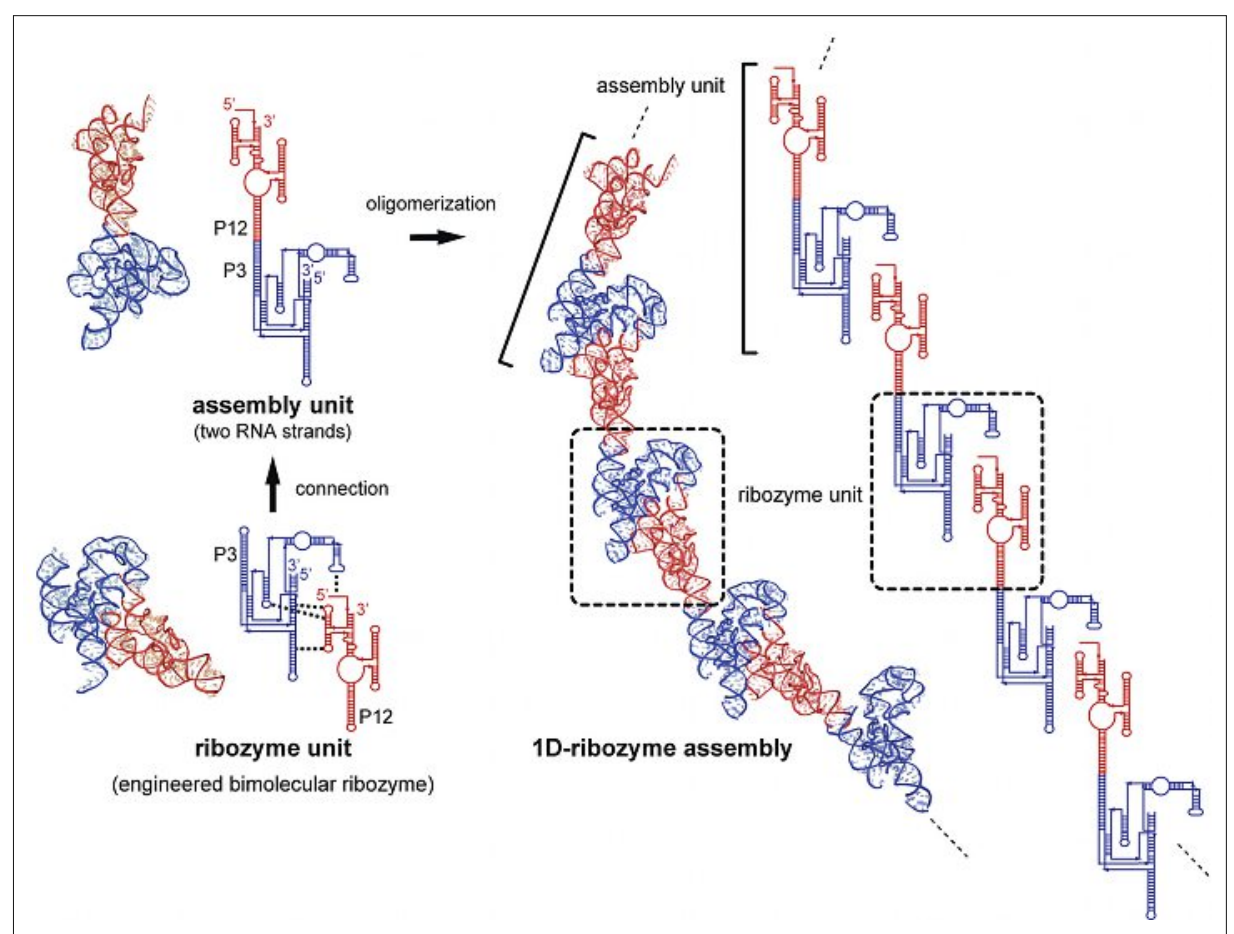

Fig. 5. A possible design of a one-dimensional (1D) ribozyme assembly, in which a bimolecular $\mathrm{R}$ Nase $\mathrm{P}$ ribozyme serves as a functional unit.

nanostructures mainly because of insufficient physical affinity of the S-domain/Cdomain complex, which seems much less stable than the bimolecular complex of the Tetrahymena ribozyme applicable to RNA nanostructure design. There are two possible approaches to resolve this problem. One approach is to engineer the current $E$. coli ribozyme to improve the physical affinity of the two domains. For this purpose, replacement of the current L9-P1 interaction with other GNRA-receptor motifs may be promising because the current receptor motif in L9 is a primitive class and has lower binding affinity and specificity than the evolved receptor motifs, including a specific receptor motif for the GAAA loop. ${ }^{[11,42]}$ The alternative approach is to examine other A-type ribozymes with high degrees of structural similarity to the $E$. coli ribozyme. The A-type ribozymes (such as the RNase P ribozymes from $T$. maritima and T. thermophilus) employed for structural studies by X-ray crystallography are good candidates for this approach. [31-33]

In addition to the application as a platform structure for designing RNA nanostructures, RNase $\mathrm{P}$ ribozyme has also been investigated as a biotechnology tool to cleave given target RNAs. The conserved mechanism of pre-tRNA recognition by RNase $\mathrm{P}$ ribozyme allows them to cleave specific mRNAs by providing an external guide sequence (EGS) RNA. ESG RNA binds to the target mRNA and forms a secondary structure that mimic the T loop and acceptor stem of pre-tRNAs. The resulting ESG-mRNA complex serves as a substrate of RNase P. With a properly designed pair of EGS RNA and target mRNA, RNase P ribozymes have been shown to break down bacterial, viral, and eukaryotic mRNAs in a catalytic manner. ${ }^{[44,45]}$

\section{Acknowledgements}

This work was supported partly by University of Toyama Discretionary Funds of the President "Toyama RNA Research Alliance" (to Y.I and S.M.).

Received: September 22, 2018

[1] T. C. King, R. Sirdeskmukh, D. Schlessinger, Microbiol. Rev. 1986, 50, 428 .

[2] K. Nakanishi, O. Nureki, Mol. Cells 2005, 19, 157.

[3] J. L. Chen, N. R. Pace, RNA 1997, 3, 557.

[4] C. Guerrier-Takada, K. Gardiner, T. Marsh, N. Pace, S. Altman, Cell 1983, 35, 849

[5] A. V. Kazantsev, N. R. Pace, Nat. Rev. Microbiol. 2006, 4, 729 .

[6] J. C. Ellis, J. W. Brown, in 'Ribonuclease P (Protein Reviews, volume 10)', F. Liu, S. Altman, Eds. Springer-Verlag New York, 2010, p. 17

[7] J. A. Pannucci, E. S. Haas, T. A. Hall, J. K. Harris, J. W. Brown, Proc. Natl. Acad. Sci. USA. 1999, 96, 7803

[8] D. Evans, S. M. Marquez, N. R. Pace, Trends Biochem. Sci. 2006, 31, 333.

[9] E. Kikovska, S. G. Svärd, L. A. Kirsebom, Proc. Natl. Acad. Sci. USA. 2007, 104, 2062.

[10] L. Randau, I. Schröder, D. Söll, Nature 2008 , $453,120$.

[11] J. Holzmann, P. Frank, E. Löffler, K. L. Bennett, C. Gerner, W. Rossmanith, Cell 2008, $135,462$.

[12] A. Gobert, B. Gutmann, A. Taschner, M. Gößringer, J. Holzmann, R. K. Hartmann, W. Rossmanith, P. Giegé, Nat. Struct. Mol. Biol. 2010, 17, 740 .

[13] B. P. Klemm, N. Wu, Y. Chen, X. Liu, K. J. Kaitany, M. J. Howard, C. A. Fierke,
Biomolecules 2016, 6, pii: E27. doi: 10.3390/ biom6020027.

[14] A. I. Nickel, N. B. Wäber, M. Gößringer, M Lechner, U. Linne, U. Toth, W. Rossmanith, R. K. Hartmann, Proc. Natl. Acad. Sci. USA. 2017, 114, 11121.

[15] E. Seif, A. Cadieux, B. F. Lang, RNA 2006, 12 1661.

[16] T. Stams, S. Niranjanakumari, C. A. Fierke, D W. Christianson, Science 1998, 280, 752.

[17] N. J. Reiter, A. Osterman, A. Torres-Larios, K. K. Swinger, T. Pan, A. Mondragón, Nature 2010, 468, 784

[18] T. A. Steitz, J. A. Steitz, Proc. Natl. Acad. Sci. USA. 1993, 90, 6498

[19] T. Pan, Biochemistry 1995, 34, 902.

[20] A. Loria, T. Pan, RNA 1996, 2, 551.

[21] Y. Ikawa, H. Shiraishi, T. Inoue, Nat. Struct. Biol. 2000, 7, 1032.

[22] S. A. Strobel, L. Ortoleva-Donnelly, Chem Biol. 1999, 6, 153.

[23] A. Torres-Larios, K. K. Swinger, T. Pan, A Mondragón, Curr. Opin. Struct. Biol. 2006, 16, 327.

[24] D. S. Waugh, C. J. Green, N. R. Pace, Science 1989, 244, 1569 .

[25] R. W. Siegel, A. B. Banta, E. S. Haas, J. W Brown, N. R. Pace, RNA 1996, 2, 452.

[26] L. B. Lai, P. P. Chan, A. E. Cozen, D. L. Bernick, J. W. Brown, V. Gopalan, T. M. Lowe, Proc. Natl. Acad. Sci. USA. 2010, 107, 22493.

[27] C. J. Green, R. Rivera-León, B. S. Vold, Nucleic Acids Res. 1996, 24, 1497.

[28] G. Mao, A. S. Srivastava, S. Wu, D. Kosek, M Lindell, L. A. Kirsebom, PLoS One 2018, 13, e0192873. doi: 10.1371/journal.pone.0192873.

[29] T. Pan, M. Jakacka, EMBO J. 1996, 15, 2249.

[30] A. S. Krasilnikov, X. Yang, T. Pan, A Mondragón, Nature 2003, 421, 760.

[31] A. S. Krasilnikov, Y. Xiao, T. Pan, A. Mondragón, Science 2004, 306, 104.

[32] A. Torres-Larios, K. K. Swinger, A. S. Krasilnikov, T. Pan, A. Mondragón, Nature 2005, 437, 584.

[33] A. V. Kazantsev, A. A. Krivenko, D. J. Harrington, S. R. Holbrook, P. D. Adams, N. R. Pace, Proc. Natl. Acad. Sci. USA. 2005, 102, 13392

[34] H. Oi, D. Fujita, Y. Suzuki, H. Sugiyama, M Endo, S. Matsumura, Y. Ikawa, J. Biochem. 2017, 161, 451 .

[35] M. M. Rahman, S. Matsumura, Y. Ikawa, J. Mol. Evol. 2018, 86, 431

[36] G. van der Horst, A. Christian, T. Inoue, Proc. Natl. Acad. Sci. USA. 1991, 88, 184.

[37] J. A. Doudna, T. R. Cech, RNA 1995, 1, 36

[38] Y. Ikawa, T. Inoue, J. Biochem. 2003, 133, 189.

[39] E. A. Doherty, D. Herschlag, J. A. Doudna, Biochemistry 1999, 38, 2982.

[40] E. S. Haas, D. P. Morse, J. W. Brown, F. J. Schmidt, N. R. Pace, Science 1991, 254, 853.

[41] M. Costa, F. Michel, EMBO J. 1997, 16, 3289.

[42] J. Ishikawa, Y. Fujita, Y. Maeda, H. Furuta, Y. Ikawa, Methods 2011, 54, 226.

[43] T. Tanaka, H. Furuta, Y. Ikawa, J. Biosci. Bioeng. 2014, 117, 407.

[44] K. Kim, F. Liu, Biochim. Biophys. Acta 2007, $1769,603$.

[45] A.J. Sawyer, D. Wesolowski, N. Gandotra, A. Stojadinovic, M. Izadjoo, S. Altman, T.R. Kyriakides, Int. J. Pharm. 2013, 453, 651.

[46] T. Ando, T. Tanaka, Y. Hori, E. Sakai, Y Kikuchi, Biosci. Biotechnol. Biochem. 2001, 65, 2798. 